\title{
INVERSE LIMITS AND ABSOLUTES OF $H$-CLOSED SPACES
}

\author{
T. O. VINSON, JR. AND R. F. DICKMAN, JR.
}

\begin{abstract}
The inverse limit of an inverse system of nonempty (respectively, minimal Hausdorff) $H$-closed spaces with continuous open surjective bonding maps is shown to be a nonempty $H$-closed (respectively, minimal Hausdorff) space. This result is used to resolve a conjecture, in the affirmative, of S. W. Willard: Every $\boldsymbol{H}$-closed space is the continuous and open image of a minimal Hausdorff space.
\end{abstract}

1. Introduction. A space (all spaces in this paper are assumed to be Hausdorff spaces) is $\mathrm{H}$-closed (HC) or absolutely closed if it is closed in any Hausdorff space in which it is embedded. A space is minimal Hausdorff (MH) if no proper subtopology is Hausdorff. The continuous image of a $\mathbf{M H}$ space is known to be HC; S. W. Willard [W, p. 270] has conjectured that, conversely, every $\mathrm{HC}$ space is the continuous image of some $\mathrm{MH}$ space. In this paper we answer Willard's conjecture affirmatively by constructing the required $\mathrm{MH}$ space using inverse limit techniques. We also show that the inverse limit of an inverse system of nonempty $\mathrm{HC}(\mathrm{MH})$ spaces with open continuous surjective bonding maps is nonempty and $\mathrm{HC}(\mathrm{MH})$. This result is the principal tool employed to answer Willard's conjecture.

Our construction was motivated by the observation that for several wellknown examples of HC spaces $X$, there is an open set $U$ of $X$ such that if disjoint copies of $X$ are identified on the complement of $U$, the resultant quotient space, $(X, U)$, is a MH space which maps onto $X$ by a continuous open map. See Examples (4.1) and (4.2). Unfortunately, we have not been able to construct such an open set $U$ for an arbitrary HC space $X$. Under these circumstances, we naturally turn to inverse limits in order to "regularize" each of the open sets of $X$, one at a time.

2. Inverse limits of HC spaces. In this section we show that an inverse system of nonempty $\mathrm{HC}$ spaces with open continuous surjective bonding maps has a nonempty $\mathrm{HC}$ inverse limit. The result is obtained by showing that every such system induces an inverse system of nonempty compact spaces with continuous bonding maps with a nonempty compact inverse limit. We also show that the inverse limit of a system of nonempty $\mathrm{MH}$ spaces with open continuous surjective bonding maps is nonempty and $\mathrm{MH}$.

(2.1) Notation and Definitions. An open filterbase on a space $X$ is a collection $\mathscr{Q}$ of nonempty open subsets of $X$ such that if $U_{1}, U_{2} \in \mathcal{Q}$, then

Received by the editors May 16, 1975 and, in revised form, February 14, 1977.

AMS (MOS) subject classifications (1970). Primary 54D25, 54D30; Secondary 54B25. 
$U_{1} \cap U_{2}$ contains an element of $\mathcal{U}$. An open filter is an open filterbase $\mathscr{U}$ such that $U_{1}, U_{2} \in \mathcal{Q}$ imply $U_{1} \cap U_{2} \in \mathcal{Q}$, and $U \in \mathcal{Q}, U \subset W, W$ open in $X$ imply $W \in \mathcal{Q}$. An open ultrafilter is an open filter properly contained in no open filter. Denote by $\mathscr{T}(p)$ the open filter of neighborhoods of $p$ (when the containing space is understood). If $\mathscr{U}$ is an open filterbase on $X$ and $p \in X$, we say $\mathscr{Q}$ converges to $p$ and write $\mathscr{U} \rightarrow p$ if each $N \in \mathscr{T}(p)$ contains some $U \in \mathcal{Q}$. A function $f: X \rightarrow Y$ is $\theta$-continuous if for every $x \in X$ and open set $V$ containing $f(x)$, there is an open set $U$ containing $x$ such that $f(\bar{U}) \subset \bar{V}$.

The space $\theta X$ of all open ultrafilters on $X$ is topologized as follows: For $U$ open in $X$ let $O_{U}$ denote the set of all open ultrafilters on $X$ containing $U$; the sets $O_{U}$ form a basis for $\theta X$. It is known that $\theta X$ is a compact, extremally disconnected Hausdorff space [IF, p. 40]. The space $\theta X$ is sometimes referred to as the hyperabsolute of $X$; the subspace $\omega X$ of $\theta X$ consisting of the convergent elements is called the absolute of $X$. (Thus, since a space is HC if and only if every open ultrafilter converges, $X$ is $\mathrm{HC}$ if and only if $\theta X=\omega X$.) Iliadis [I, pp. 295-296] has shown that $\omega X$ maps irreducibly, $\theta$-continuously and perfectly onto $X$.

Let $\mathbb{Q}$ be a set directed by $<$. Suppose for each $\alpha \in \mathbb{Q}$ that $X_{\alpha}$ is a space and that for each pair $\alpha, \beta \in \mathbb{Q}$ with $\beta \leqslant \alpha$, there is a continuous function $\Phi_{\alpha \beta}: X_{\alpha} \rightarrow X_{\beta}$ such that $\Phi_{\alpha \alpha}$ is the identity map on $X_{\alpha}$ and $\gamma \leqslant \beta \leqslant \alpha$ implies $\Phi_{\alpha \gamma}=\Phi_{\beta \gamma} \Phi_{\alpha \beta}$. Then we say $\left\{X_{\alpha}, \Phi_{\alpha \beta}, \mathbb{Q}\right\}$ is an inverse system. A point $\left(p_{\alpha}\right) \in \Pi_{\alpha \in \mathbb{Q}} X_{\alpha}$ is called a thread of the system if $\beta \leqslant \delta$ implies $p_{\beta}=\Phi_{\delta \beta}\left(p_{\delta}\right)$. The set of all threads is called the inverse limit of the system and is denoted by inv $\lim \left\{X_{\alpha}, \Phi_{\alpha \beta}, \mathbb{Q}\right\}$ or simply by $X_{\infty}$. If $B \subset X_{\beta}$ for some $\beta \in \mathbb{Q}$, denote by $\langle B\rangle_{\infty}$ the set $\left\{\left(p_{\alpha}\right) \in X_{\infty} \mid p_{\beta} \in B\right\}$.

(2.2) If $X$ is $H C$, then the natural map $e: \theta X \rightarrow X$ sending an open ultrafilter to the point to which it converges is a $\theta$-continuous perfect surjection [I, p. 296].

(2.3) Let $\left\{X_{\alpha}, \Phi_{\alpha \beta}, \mathbb{Q}\right\}$ be an inverse system. Sets of the form $\langle V\rangle_{\infty}$, where $V$ is open in some $X_{\beta}$, constitute a basis for $X_{\infty}$ [C, p. 234].

(2.4) If $\left\{X_{\alpha}, \Phi_{\alpha \beta}, \mathbb{Q}\right\}$ is an inverse system of nonempty compact spaces and continuous functions, then its inverse limit is nonempty and compact [C, $p$. 235].

(2.5) Let $f: X \rightarrow Y$ be a continuous open surjection of $H C$ spaces. Let $p \in X$, $q=f(p)$, and let $\mathcal{U}^{\prime}$ be an open ultrafilter on $Y$ converging to $q$. Then there exists $\mathscr{U} \in \theta X$ with $\mathscr{U} \rightarrow p$ and $f(\mathcal{U})=\mathcal{U}^{\prime}$ (that is, $\mathcal{U}^{\prime}=\{f(U) \mid U \in \mathcal{U}\}$ ) [M, p. 290].

(2.6) Let $f: X \rightarrow Y$ be a continuous open surjection of HC spaces. Then there exists a unique continuous open surjection $f^{*}: \theta X \rightarrow \theta Y$ such that the diagram below commutes:

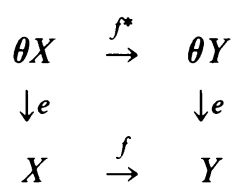

Proof. For any open ultrafilter $\mathscr{U}$ on $X$, define $f^{*}(\mathscr{U})=\mathscr{U}^{\prime}=\{f(U) \mid U$ 
$\in \mathscr{U}$ \}. Then since $f$ is open and continuous, $f^{*}(\mathcal{U})$ is an open ultrafilter on $Y$ for any $\mathcal{U} \in \theta X$. To see that $f^{*}$ is continuous, let $O_{U}=\left\{\mathcal{U}^{\prime} \mid U \in \mathcal{U}^{\prime}\right\}$ be a basic open set in $\theta Y$. One sees that $f^{*-1}\left(O_{U}\right)=O_{f^{-1}(U)}$ which is open in $\theta X$. Also, $f^{*}$ is open, since $f^{*}\left(O_{U}\right)=O_{f(U)}$. By (2.5), $f^{*}$ is surjective. Since $\mathcal{Q} \rightarrow p$ implies $f(U) \rightarrow f(p), f e=e f^{*}$ and the diagram commutes. Finally, since $f^{*}\left(O_{U}\right)=O_{f(U)}$, we see that $f^{*}$ is the unique map satisfying the required conditions.

(2.7) If $\left\{X_{\alpha}, \Phi_{\alpha \beta}, Q\right\}$ is an inverse system with open maps $\Phi_{\alpha \beta}$, and if $U$ is open in $X_{\beta}, \beta \in \mathbb{Q}$, then $\overline{\langle U\rangle_{\infty}}=\langle\bar{U}\rangle_{\infty}$.

Proof. Suppose $\left(p_{\alpha}\right) \notin \overline{\langle U\rangle_{\infty}}$. Then there is some open set $V$ in some $X_{\gamma}$ with $\left(p_{\alpha}\right) \in\langle V\rangle_{\infty}$ and $\langle U\rangle_{\infty} \cap\langle V\rangle_{\infty}=\varnothing$. Let $\delta>\beta$ and $\delta>\gamma$, and let $W_{1}=\Phi_{\delta \beta}^{-1}(U)$ and $W_{2}=\Phi_{\delta \gamma}^{-1}(V)$. Observe that $\langle U\rangle_{\infty}=\left\langle W_{1}\right\rangle_{\infty}$ and $\langle V\rangle_{\infty}$ $=\left\langle W_{2}\right\rangle_{\infty}$, so $W_{1} \cap W_{2}=\varnothing$. Since $p_{\delta} \in W_{2}, p_{\beta} \in \Phi_{\delta \beta}\left(W_{2}\right)$, which is an open set missing $U$. Thus $\left(\underline{p}_{\alpha}\right) \notin\langle\bar{U}\rangle_{\infty}$.

Conversely, if $\left(p_{\alpha}\right) \notin\langle\bar{U}\rangle_{\infty}$, then $p_{\beta} \notin \bar{U}$, so $\left(p_{\alpha}\right) \in\left\langle X_{\beta}-\bar{U}\right\rangle_{\infty}$, an open set missing $\langle U\rangle_{\infty}$. Thus $\left(p_{\alpha}\right) \notin \overline{\langle U\rangle_{\infty}}$.

(2.8) THEOREM. The inverse limit of an inverse system of nonempty HC spaces with continuous open surjective bonding maps is a nonempty HC space.

Proof. Let $\left\{X_{\alpha}, \Phi_{\alpha \beta}, Q\right\}$ be an inverse system of nonempty HC spaces and open surjections. For each pair $\alpha, \beta \in \mathbb{Q}, \beta<\alpha$, let $\Phi_{\alpha \beta}^{*}$ be the unique continuous open surjection of $\theta X_{\alpha}$ onto $\theta X_{\beta}$ given by (2.6). Note that since $\Phi_{\alpha \beta}^{*}$ is the unique map satisfying (2.6), $\left\{\theta X_{\alpha}, \Phi_{\alpha \beta}^{*}, \mathcal{Q}\right\}$ is an inverse system, i.e. if $\delta<\beta<\alpha$, then $\Phi_{\beta \delta}^{*} \Phi_{\alpha \beta}^{*}=\Phi_{\alpha \delta}^{*}$. Hence, by (2.4), the inverse limit $X^{*}$ of $\left\{\theta X_{\alpha}: \Phi_{\alpha \beta}^{*}, Q\right\}$ is a nonempty compact Hausdorff space. We will show that the map $e_{\infty}: X^{*} \rightarrow X_{\infty}$, which assigns to each thread of ultrafilters the thread of their limits, is surjective and $\theta$-continuous. To this end, let $\beta \in \mathbb{Q}$ and let $V$ be open in $X_{\beta}$. We will show $e_{\infty} \overline{\left(\left\langle O_{V}\right\rangle_{\infty}\right)} \subset \overline{\langle V\rangle_{\infty}}$, which equals $\langle\bar{V}\rangle_{\infty}$ by (2.7). Suppose $e_{\infty}\left(\left(\mathcal{U}_{\alpha}\right)\right) \notin\langle\bar{V}\rangle_{\infty}$. Then no point of $\bar{V}$ is an adherent point of $\mathscr{U}_{\beta}$, which implies $X_{\beta}-\bar{V} \equiv W \in \mathcal{U}_{\beta}$. So $\mathcal{Q}_{\beta} \in O_{W}$ and $\left(\mathcal{U}_{\alpha}\right) \in\left\langle O_{W}\right\rangle_{\infty}$. But $\left\langle O_{W}\right\rangle_{\infty} \cap\left\langle O_{V}\right\rangle_{\infty}=\varnothing$, so $\left(\mathcal{U}_{\alpha}\right) \notin\left\langle O_{V}\right\rangle_{\infty}$, and $e_{\infty}$ is $\theta$-continuous.

Now let $\left(p_{\alpha}\right) \in X_{\infty}$. Let $e_{\alpha}: \theta X_{\alpha} \rightarrow X_{\alpha}$ be the $\theta$-continuous evaluation function of (2.2). By (3.4) of [DP], $P_{\alpha} \equiv e_{\alpha}^{-1}\left(p_{\alpha}\right)$ is compact (and nonempty), and by (2.6), $\left\{P_{\alpha}, \Phi_{\alpha \beta}^{*} \mid P_{\alpha}, \mathbb{Q}\right\}$ is an inverse system. Its inverse limit is nonempty by (2.4) and is a subset of $e_{\infty}^{-1}\left(\left(p_{\alpha}\right)\right)$. Thus $e_{\infty}$ is surjective and $X_{\infty}$ is nonempty. Finally, $X_{\infty}$ is $\mathrm{HC}$ since it is the $\theta$-continuous image of the compact space $X^{*}$.

(2.9) Let $\left\{X_{\alpha}, \Phi_{\alpha \beta}, Q\right\}$ be an inverse system with surjective open bonding maps and surjective projections. If $\beta \in \mathbb{Q}$ and $U$ is regular open in $X_{\beta}$, then $\langle U\rangle_{\infty}$ is regular open in $X_{\infty}$.

Proof. By (2.7), $\overline{\langle U\rangle_{\infty}}=\langle\bar{U}\rangle_{\infty}$. Suppose $\left(p_{\alpha}\right) \in \operatorname{int}\langle\bar{U}\rangle_{\infty}$. Then $\left(p_{\alpha}\right) \in$ $\langle V\rangle_{\infty} \subset\langle\bar{U}\rangle_{\infty}$ for some $V$ open in some $X_{\gamma}$. Let $\delta>\gamma$ and $\delta>\beta$. Now since $\Phi_{\delta \gamma}$ is surjective, $\left\langle\Phi_{\delta \gamma}^{-1}(V)\right\rangle_{\infty}=\langle V\rangle_{\infty}$; also since the projections are surjective and $\left\langle\Phi_{\delta \gamma}^{-1}(V)\right\rangle_{\infty} \subseteq\langle\bar{U}\rangle_{\infty}, \Phi_{\delta \beta} \Phi_{\delta \gamma}^{-1}(V) \subseteq \bar{U}$. Thus $p_{\beta} \in \Phi_{\delta \beta} \Phi_{\delta \gamma}^{-1}(V)$ 
which, being open in $\bar{U}$, lies in $U$. Thus $\left(p_{\alpha}\right) \in\left\langle\Phi_{\delta \beta} \Phi_{\delta \gamma}^{-1}(V)\right\rangle_{\infty} \subset\langle U\rangle_{\infty}$, so $\operatorname{int}\langle\bar{U}\rangle_{\infty} \subset\langle U\rangle_{\infty}$, and $\langle U\rangle_{\infty}$ is regular open.

(2.10) Let $\left\{X_{\alpha}, \Phi_{\alpha \beta}, \mathbb{Q}\right\}$ be an inverse system of nonempty compact sets and continuous surjections, let $\gamma \in \mathbb{Q}$, and let $p \in X_{\gamma}$. Then $\langle\{p\}\rangle_{\infty} \neq \varnothing$.

Proof. Let $\delta \in \mathbb{Q}$ and let $\gamma, \delta<\varepsilon$. Put $P_{\delta}=\Phi_{\varepsilon \delta} \Phi_{\varepsilon \gamma}^{-1}(p)$. One sees that $P_{\delta}$ is well defined (i.e., independent of the choice of $\varepsilon$ ) and that $\left\{P_{\alpha}, \Phi_{\alpha \beta} \mid P_{\alpha}, \mathbb{Q}\right\}$ is an inverse system of nonempty sets. Thus by (2.4), $P_{\infty} \neq \varnothing$. By our selection of $P_{\delta}, P_{\infty}=\langle\{p\}\rangle_{\infty}$ and the proof is complete.

(2.11) Let $\left\{X_{\alpha}, \Phi_{\alpha \beta}, \mathbb{Q}\right\}$ be an inverse system of nonempty $H C$ spaces and continuous open surjections. Let $\gamma \in \mathbb{Q}$ and $p \in X_{\gamma}$. Then $\langle\{p\}\rangle_{\infty} \neq \varnothing$.

Proof. Let $\left\{\theta X_{\alpha}, \Phi_{\alpha \beta}^{*}, \mathbb{Q}\right\}$ be the induced system of (2.6). Let $\dot{U}$ be any open ultrafilter on $X_{\gamma}$ converging to $p$. By $(2.10),\langle\{U\}\rangle_{\infty} \neq \varnothing$ (in the induced system) and so $\varnothing \neq e_{\infty}\left(\langle\{U\}\rangle_{\infty}\right) \subset\langle\{p\}\rangle_{\infty}$.

(2.12) ThEOREM. Let $\left\{X_{\alpha}, \Phi_{\alpha \beta}, \mathbb{Q}\right\}$ be an inverse system of $M H$ spaces and continuous open surjections. Then $X_{\infty}$ is $M H$.

Proof. It is known that a space is MH if and only if it is $\mathrm{HC}$ and semiregular [K]. By (2.8), $X_{\infty}$ is HC. It remains to show that $X_{\infty}$ is semiregular. Let $\left(p_{\alpha}\right) \in X_{\infty}$ and let $\langle V\rangle_{\infty}$ be a neighborhood of $\left(p_{\alpha}\right)$, where $V$ is open in some $X_{\gamma}$. Since $X_{\gamma}$ is $\mathrm{MH}$, there exists a regular open set $W$ such that $p_{\gamma} \in W \subset V$. We assert that $\langle W\rangle_{\infty}$ is regular open in $X_{\infty}$ : Let $\left(q_{\alpha}\right) \in \operatorname{int} \overline{\langle W\rangle_{\infty}}$. Then $\left(q_{\alpha}\right) \in\langle Z\rangle_{\infty} \subset \overline{\langle W\rangle_{\infty}}$ for some $Z$, open in some $X_{\delta}$ and, by (2.7), $\langle Z\rangle_{\infty} \subset\langle\bar{W}\rangle_{\infty}$. Let $\gamma, \delta\left\langle\varepsilon\right.$. Then $\langle Z\rangle_{\infty}=\left\langle\Phi_{e \delta}^{-1}(Z)\right\rangle_{\infty} \subset$ $\langle\bar{W}\rangle_{\infty}$ implies that $Z_{\gamma} \equiv \Phi_{e \gamma} \Phi_{e \gamma}^{-1}(Z) \subset \bar{W}$, for by (2.11), every $p \in \Phi_{e \delta}^{-1}(Z)$ is the coordinate of some thread in $X_{\infty}$; such a thread must have $\gamma$ th coordinate in $\bar{W}$, so $\Phi_{\text {ey }}(p) \in \bar{W}$. Thus the open set $Z_{\gamma}$ contains $P_{\gamma}$ and is contained in $\bar{W}$. Since $W$ is regular open, we have $Z_{\gamma} \subset W$. Now $q_{\gamma} \in Z \subset W$, so $\left(q_{\alpha}\right) \in\langle W\rangle_{\infty}$. We have shown that int $\overline{\langle W\rangle_{\infty}} \subset\langle W\rangle_{\infty}$; thus $\langle W\rangle_{\infty}$ is a regular open set in $X_{\infty}$ containing $\left(p_{\alpha}\right)$ and lying entirely in $\langle V\rangle_{\infty}$. This completes the proof.

In (4.4) we give an example of a system of $\mathrm{MH}$ spaces with continuous surjection bonding maps with a nonminimal Hausdorff inverse limit.

3. HC spaces as the continuous open image of MH spaces. In this section, for any HC space $X$, we use Theorem (2.8) and construct an inverse system of HC spaces with continuous open surjective maps and with a MH inverse limit $X_{\infty}$ that maps onto $X$. We first show that it is possible to "regularize" an open set $U$ of $X$ while retaining the $H$-closedness of $X$, i.e., we construct an HC space $Z$ containing $X$ in which $U$ is a regular open subset of $Z$.

(3.1) The regularization of an open set. Let $U$ be open in a space $X$ and let $h$ be a homeomorphism of $X$ onto $X^{\prime}$ with $X \cap X^{\prime}=\varnothing$. Let $\psi$ be the natural map on $X \cup X^{\prime}$ for the decomposition whose only nondegenerate elements are doubletons of the form $\{x, h(x)\}$ with $x \in X-U$. Denote by $(X, U)$ the decomposition space with the quotient topology. Let $P: X \cup X^{\prime} \rightarrow X$ be 
given by $P(x)=x, x \in X$, and $P(x)=h^{-1}(x), x \in X^{\prime} . P$ is easily seen to be continuous and open, and $P \psi^{-1} \equiv \pi$ is single-valued, continuous, open, and surjective. We call $\pi$ the natural projection of $(X, U)$ onto $X$.

One may think of $(X, U)$ as the union of $X$ with a disjoint copy $U^{\prime}$ of $U$ so that the frontiers of $U$ and $U^{\prime}$ coincide. Then $U^{\prime}$ forms a "blister" on $X$ over $U$, causing both $U$ and $U^{\prime}$ to be regular open in $(X, U)$. Specifically,

(3.2) $U$ and $h(U)$ are regular open in $(X, U)$.

Proof. Let $p \in\left[\operatorname{cl}_{(X, U)} U\right]-U$. Since $U$ and $h(U)$ are disjoint open in $(X, U), p \notin h(U)$, so $p=\{q, h(q)\}$ for some $q \in X-U$. If $W$ is open about $p$, then $\psi^{-1}(W)$ is open about $\{q, h(q)\}$ in $X \cup X^{\prime}$. In particular, $\psi^{-1}(W)$ intersects $h(U)$, so $p \notin$ int $\operatorname{cl}_{(X, U)} U$. Therefore $U$ is regular open, and similarly, so is $h(U)$.

(3.3) If $X$ is $H C$ and $U \subset X$ is open, then $(X, U)$ is $H C$.

Proof. This follows immediately since $(X, U)$ is the continuous image of the HC space $X \cup X^{\prime}$.

To follow is a process in which the open sets of $X$ are allowed to "blister" one at a time, cumulatively. The resulting inverse limit space will be semiregular and will map openly onto $X$.

(3.4) Let $S$ be a well-ordered set with no supremum. Then there is a well-ordered set $T$ and a surjective function $G: T \rightarrow S$ with cofinal point inverses.

Proof. Let $T=S \times S$ be ordered by: $(a, b)<(c, d)$ if $a<c$ or if $a=c$ and $b<d$. Then $T$ is well ordered. Put $G(a, b)=b$. Then $G^{-1}(b)=$ $\{(a, b) \mid a \in S\}$ which is cofinal in $T$.

(3.5) Construction. Let $X_{1}$ be $\mathrm{HC}$ with topology $\mathscr{B}$. Let $\mathscr{B}$ be well ordered with no supremum. By (3.4) there is a well-ordered set $\mathcal{Q}$ and a surjective indexing function $i: \mathbb{Q} \rightarrow \mathscr{B}$ with cofinal point inverses. Denote by $U_{\alpha}$ the image $i(\alpha)$. Then for each $B \in \mathscr{B}$ and $\alpha \in \mathbb{Q}$ there exists $\beta>\alpha$ such that $B=U_{\beta}$. We define an inverse system $\left\{X_{\alpha}, \Phi_{\alpha \beta}, \mathbb{Q}\right\}$ inductively as follows: let $X_{2}=\left(X_{1}, U_{1}\right)$ and let $\Phi_{21}: X_{2} \rightarrow X_{1}$ be the natural projection. Suppose for $\delta \leqslant \gamma \leqslant \beta<\alpha$ that $X_{\beta}$ and $\Phi_{\beta \gamma}: X_{\beta} \rightarrow X_{\gamma}$ are defined with $X_{\beta}$ HC, $\Phi_{\beta \gamma}$ continuous, open and surjective, and $\Phi_{\beta \delta}=\Phi_{\gamma \delta} \Phi_{\beta \gamma}$. For $\alpha \in \mathbb{Q}$, denote by $\mathbb{Q}_{\alpha}$ the set $\{\beta \in \mathbb{Q} \mid \beta<\alpha\}$. We distinguish two cases. If $\alpha$ is a limit ordinal, let $X_{\alpha}=\operatorname{inv} \lim \left\{X_{\beta}, \Phi_{\beta \gamma}, \mathbb{Q}_{\alpha}\right\}$. Let $\Phi_{\alpha \beta}: X_{\alpha} \rightarrow X_{\beta}$ be the projection onto the $\beta$ th coordinate. If $\alpha$ is not a limit ordinal, let $X_{\alpha}=$ $\left(X_{\alpha-1}, \Phi_{(\alpha-1) 1}^{-1}\left(U_{\alpha-1}\right)\right)$ and let $\Phi_{\alpha(\alpha-1)}: X_{\alpha} \rightarrow X_{\alpha-1}$ be the natural projection with $\Phi_{\alpha \beta}=\Phi_{(\alpha-1) \beta} \Phi_{\alpha(\alpha-1)}$.

For $\beta \leqslant \alpha$ let $\Phi_{\alpha \beta}^{*}: \theta X_{\alpha} \rightarrow \theta X_{\beta}$ be the induced map of (2.6). Let

$$
X^{*}=\text { inv } \lim \left\{\theta X_{\alpha}, \Phi_{\alpha \beta}, Q\right\} \text {. }
$$

Let $e_{\alpha}: \theta X_{\alpha} \rightarrow X_{\alpha}$ and $e_{\infty}: X^{*} \rightarrow X_{\infty}$ be the evaluation maps of (2.6) and (2.8).

Notation. Unless otherwise defined, objects referred to in the balance of this section are those of the above construction.

(3.6) The maps $\Phi_{\alpha \beta}$ are continuous open surjections. 
Proof. Suppose $\Phi_{\beta \gamma}$ is a continuous open surjection for all $\gamma \leqslant \beta<\alpha$.

Case 1. $\alpha$ is a limit ordinal.

We make the inductive assumption that for $\beta<\alpha$ and $p_{\beta} \in X_{\beta}$, there is a thread $\left(p_{t}\right), 1 \leqslant t \leqslant \beta$, so that if $1 \leqslant s \leqslant t \leqslant \beta$, then the consistency property $\Phi_{t s}\left(p_{t}\right)=p_{s}$ holds. Let $\beta \in \mathbb{Q}$ and $p_{\beta} \in X_{\beta}$. Thus, for each $1 \leqslant t \leqslant$ $\beta$, assume we have chosen $p_{t} \in X_{t}$ so that the thread $\left(p_{t}\right)$ has the above consistency property.

Suppose $\beta<\delta<\alpha$ and for all $\beta \leqslant \gamma<\delta$ we have chosen $p_{\gamma} \in X_{\gamma}$ so that the thread $\left(p_{t}\right), 1 \leqslant t \leqslant \gamma$, is a thread with the consistency property. If $\delta=\delta_{0}+1$, just choose $p_{\delta} \in X_{\delta}$ so that $\Phi_{\delta \delta_{0}}\left(p_{\delta}\right)=p_{\delta_{0}}$. If $\delta$ is a limit ordinal, let $p_{\delta}=\left(p_{t}\right), 0 \leqslant t<\delta$. Finally, $p_{\alpha}$ is defined to be $\left(p_{t}\right), 0 \leqslant t<\alpha$. Therefore each $\Phi_{\alpha \beta}$ is a surjection.

For $B \subset X_{\beta}, \beta<\alpha$, define $\langle B\rangle_{\alpha}=\left\{\left(p_{\delta}\right) \in X_{\alpha} \mid p_{\beta} \in B\right\}$. If $U$ is open in $X_{\beta}$, then $\Phi_{\alpha \beta}^{-1}(U)=\langle U\rangle_{\alpha}$ which is basic open in $X_{\alpha}$ by (2.3), so $\Phi_{\alpha \beta}$ is continuous. Conversely, if $\langle U\rangle_{\alpha}$ is basic open in $X_{\alpha}$, where $U \subset X_{\delta}$ for some $\delta \in \mathbb{Q}_{\alpha}$, then $\Phi_{\alpha \beta}\left(\langle U\rangle_{\alpha}\right)=\Phi_{\beta \delta}^{-1}(U)$ or $\Phi_{\delta \beta}(U)$ according as $\delta<\beta$ or $\beta \leqslant \delta$, open in either case. Thus $\Phi_{\alpha \beta}$ is open.

Continuity comes from the fact that the functions are coordinate projections from the inverse limit.

Case 2. $\alpha$ is not a limit ordinal. $\Phi_{\alpha(\alpha-1)}$ is a continuous open surjection as noted earlier, as is $\Phi_{(\alpha-1) \beta}$ by inductive hypothesis. Thus so is $\Phi_{\alpha \beta}=$ $\Phi_{(\alpha-1) \beta} \Phi_{\alpha(\alpha-1)}$.

(3.7) The projection $\Pi: X_{\infty} \rightarrow X_{1}$ is a continuous open surjection.

Proof. Apply the argument of Case 1 above.

(3.8) $X_{\alpha}$ is $H C$ for each $\alpha \in \mathbb{Q}$.

Proof. Suppose true for $\beta<\alpha$. If $\alpha$ is not a limit ordinal, then $X_{\alpha}=$ $\left(X_{\alpha-1}, \Phi_{(\alpha-1) 1}^{-1}\left(U_{\alpha-1}\right)\right)$ which is $\mathrm{HC}$ by (3.3). Otherwise

$$
X_{\alpha}=\operatorname{inv} \lim \left\{X_{\beta}, \Phi_{\beta \gamma}, \mathbb{Q}_{\alpha}\right\}
$$

which is $\mathrm{HC}$ by (2.8).

(3.9) $X_{\infty}$ is semiregular.

Proof. Let $\left(p_{\alpha}\right) \in X_{\infty}$ and let $R$ be an open subset of $X_{\infty}$ containing $\left(p_{\alpha}\right)$. By (2.3) there exists $\beta \in \mathbb{Q}$ and $V$ open in $X_{\beta}$ such that $\left(p_{\alpha}\right) \in\langle V\rangle_{\infty} \subset R$. Now $\varphi_{\beta 1}(V) \in B$ and, as seen in (3.5), we may assume that $\varphi_{\beta 1}(V)=U_{\gamma}$ where $\gamma>\beta$. Note that since the bonding maps are surjections,

$$
\begin{aligned}
\langle V\rangle_{\infty} & =\left\langle\varphi_{\gamma \beta}^{-1}(V)\right\rangle_{\infty}=\left\langle\varphi_{\gamma 1}^{-1} \varphi_{\beta 1}(V)\right\rangle_{\infty}=\left\langle\varphi_{\gamma 1}^{-1}\left(U_{\gamma}\right)\right\rangle_{\infty} \\
& =\left\langle\varphi_{(\gamma+1) \gamma}^{-1}\left[\varphi_{\gamma 1}^{-1}\left(U_{\gamma}\right)\right]\right\rangle_{\infty} .
\end{aligned}
$$

Now by (3.2), $\varphi_{(\gamma+1) \gamma}^{-1}\left[\varphi_{\gamma 1}^{-1}\left(U_{\gamma}\right)\right]$ is the union of two disjoint regular open sets. Let $T$ be the one containing $p_{\gamma+1}$. Then by (2.9), $\langle T\rangle_{\infty}$ is regular open and $\left(p_{\alpha}\right) \in\langle T\rangle_{\infty} \subset\langle V\rangle_{\infty} \subset R$ and $X_{\infty}$ is semiregular.

Thus we have proved the following:

(3.10) THEOREM. Every $H C$ space is the continuous open image of a $\mathrm{MH}$ sfrce. 
Proof. $X_{\infty}$ is an HC, semiregular space that is mapped onto $X$ by the projection $\pi: X_{\infty} \rightarrow X_{1}$.

\section{Examples.}

(4.1) Let $\tilde{X}$ be the set of points in the plane of the form $a_{m n}=(1 / m, 1 / n)$ or $c_{m}=(1 / m, 0)$, where $m$ and $n$ are positive integers. Give $X$ the relative usual topology. Suppose $\alpha \notin \tilde{X}$ and let $X=\tilde{X} \cup\{\alpha\}$. Basic neighborhoods of $\alpha$ are sets of the form $\left\{\alpha_{m n} \mid n>n_{0}\right\} \cup\{\alpha\}$. Then $X$ is HC but not MH (X is not semiregular at $\alpha)$. Let $U=X-\left\{c_{m}\right\}_{m=1}^{\infty}$. One checks that $(X, U)$ is MH and maps openly onto $X$ by the natural projection.

(4.2) Let $Y$ be the closed interval $[0,1]$ and let the topology be generated by the usual one together with the set $Q$ of rational numbers in $[0,1]$. Then $Y$ is $\mathrm{HC}$ but not semiregular at any rational number. However, $(Y, Q)$ is $\mathrm{MH}$ and maps onto $Y$.

(4.3) Let $X$ be the space defined in (4.1) and let $X_{n}=X$ for $n=1,2, \ldots$ Define $\Phi_{n(n-1)}: X_{n} \rightarrow X_{n-1}$ by $a_{m n} \rightarrow a_{(m+1) n}, c_{m} \rightarrow c_{m}$, and $\alpha \rightarrow \alpha$. Consider $\left\{X_{n}, \Phi_{m n}, I_{+}\right\}$where $I_{+}$is the set of positive integers and

$$
\Phi_{m n}=\Phi_{(n+1) n} \Phi_{(n+2)(n+1)} \cdots \Phi_{m(m-1)} .
$$

The only threads in the system have the form $\left(c_{n}, c_{n}, c_{n}, \ldots\right)$ or $(\alpha, \alpha, \alpha, \ldots)$, and we see that $X_{\infty}$ is an infinite discrete space, hence not HC. Thus the requirement in (2.8) that the maps $\Phi_{\alpha \beta}$ be surjective is necessary.

(4.4) The requirement in (2.12) that $\Phi_{\alpha \beta}$ be open is necessary. For let $Y$ be the space $(X, U)$ of (4.1). We "unzip" $Y$ along $X-U$ in the following sense to get two disjoint open copies of $X$. Let $\tilde{Y}_{k}$ consist of the planar points $a_{m n}=(1 / m, 1 / n), c_{m}=(1 / m, 0)\left(m, n \in I_{+}\right), d_{m}=(1 / m,-1)(m \leqslant k)$, and $b_{m n}=(1 / m, 1 /-n-1)$ for $m \leqslant k$ and $b_{m n}=(1 / m, 1 /-n)$ for $m>$ $k$. Adjoin to $\tilde{Y}_{k}$ the two new points $\alpha, \beta$ whose basic neighborhoods have the form $\left\{a_{m n} \mid n>n_{0}\right\} \cup\{\alpha\}$ and $\left\{b_{m n} \mid n>n_{0}\right\} \cup\{\beta\}$, respectively, where $n_{0} \in$ $I_{+}$. Call this augmented space $Y_{k}$. Define $\Phi_{k(k-1)}: Y_{k} \rightarrow Y_{k-1}$ to be the identity function except that $\Phi_{k(k-1)}\left(d_{k}\right)=c_{k}$. Note that $Y_{k}$ is $\mathrm{MH}$ and $\Phi_{k(k-1)}$ is continuous and surjective but not open. For $j<k$, put $\Phi_{k j}=$ $\Phi_{(j+1) j} \cdots \Phi_{k(k-1)}$ as usual and let $\Phi_{k k}$ be the identity function on $Y_{k}$. Consider $Y_{\infty}=\operatorname{inv} \lim \left\{Y_{k}, \Phi_{k j}, I_{+}\right\}$. We observe that its elements are of the following form.

(1) $\bar{\alpha}=(\alpha, \alpha, \alpha, \ldots)$,

(2) $\bar{\beta}=(\beta, \beta, \beta, \ldots)$,

(3) $\bar{a}_{m n}=\left(a_{m n}, a_{m n}, \ldots\right)$,

(4) $\bar{b}_{m n}=\left(b_{m n}, b_{m n}, \ldots\right)$

(5) $\bar{c}_{m}=\left(c_{m}, c_{m}, c_{m}, \ldots\right)$,

(6) $\bar{d}_{m}=\left(c_{m}, c_{m}, \ldots, c_{m}, d_{m}, d_{m}, \ldots\right)\left(m-1 c_{m}\right.$ 's).

It is easy to see that $\bar{a}_{m n}$ and $\bar{b}_{m n}$ are isolated (e.g., for any $k \in I_{+}$let $a_{m n} \in Y_{k}$; since $\left\{a_{m n}\right\}$ is open in $Y_{k},\left\{\bar{a}_{m n}\right\}=\left\langle\left\{a_{m n}\right\}\right\rangle_{\infty}$ is open). Also, basic neighborhoods of $\bar{\alpha}$ and $\bar{\beta}$ are $\left\{\bar{a}_{m n} \mid n>n_{0}\right\} \cup\{\bar{\alpha}\}$ and $\left\{\bar{b}_{m n} \mid n>n_{0}\right\} \cup\{\bar{\beta}\}$, respectively. If $V$ is a basic neighborhood of $c_{m}$ in $Y_{k}$, then 


$$
V=\left\{a_{m n} \mid n>n_{0}\right\} \cup\left\{c_{m}\right\}
$$

or

$$
V=\left\{a_{m n} \mid n>n_{0}\right\} \cup\left\{b_{m n} \mid n>n_{0}\right\} \cup\left\{c_{m}\right\}
$$

according as $k \geqslant m$ or $k<m$. The former case provides the basic neighborhoods $\langle V\rangle_{\infty}$ of $\bar{c}_{m}$; namely, $\left\{\bar{a}_{m n}|n\rangle n_{0}\right\} \cup\left\{\bar{c}_{m}\right\}$. Analogously, basic neighborhoods of $\bar{d}_{m}$ are of the form $\langle W\rangle_{\infty}$, where $W$ is a basic neighborhood of $d_{m}$ in $Y_{k}, k \geqslant m$, and $\langle W\rangle_{\infty}=\left\{\bar{b}_{m n}|n\rangle n_{0}\right\} \cup\left\{\bar{d}_{m}\right\}$. Now $Y=\left\{\bar{a}_{m n}, \bar{b}_{m n}, \bar{c}_{m}, \bar{d}_{m}, \bar{\alpha}, \bar{\beta}\right\}$ is the disjoint open union of $\left\{\bar{a}_{m n}, \bar{c}_{m}, \bar{\alpha}\right\}$ and $\left\{\bar{b}_{m n}, \bar{d}_{m}, \bar{\beta}\right\}$, each copies of the space $X$ of (4.1). Hence $Y_{\infty}$ fails to be semiregular at $\bar{\alpha}$ and $\bar{\beta}$, and so is not $\mathrm{MH}$.

ADDENDUM. In a private communication, the authors have learned that $\mathrm{L}$. M. Friedler and D. H. Pettey have independently solved Willard's conjecture using inverse limit techniques. In this paper we utilized the induced system of absolutes over a system with open surjective bonding maps, while in their result, Friedler and Pettey proved that the inverse limit of a system of nonempty $\mathrm{HC}$ spaces over a directed set with a cofinal chain is nonempty and HC. It is not known whether the inverse limit of nonempty HC spaces with surjective bonding maps is nonempty and $\mathrm{HC}$.

\section{BIBLIOGRAPHY}

[B] M. P. Berri, Minimal topological spaces, Trans. Amer. Math. Soc. 108 (1963), 97-105.

[BPS] M. P. Berri, J. R. Porter and R. M. Stephenson, Jr., A survey of minimal topological spaces, Proc. Kanpur Topological Conf., 1968, pp. 93-114.

[C] C. E. Capel, Inverse limit spaces, Duke Math. J. 21 (1954), 233-245.

[DP] R. F. Dickman, Jr. and J. R. Porter, O-closed subsets of Hausdorff spaces, Pacific J. Math. 59 (1975), 407-415.

[I] S. Iliadis, Absolutes of Hausdorff spaces, Soviet Math. Dokl. 4 (1963), 295-298.

[IF] S. Iliadis and S. Fomin, The method of centered systems in the theory of topological spaces, Russian Math. Surveys 21 (1966), 37-62.

[K] M. Katetov, Uber H-abgeschlossene und bikompakte Räume, Casopis Pest. Mat. 69 (1940), $36-49$.

[L] C. T. Liu, Absolutely closed spaces, Trans. Amer. Math. Soc. 130 (1968), 86-104.

[M] E. Michael, Bi-quotient maps and cartesian products of quotient maps, Ann. Inst. Fourier (Grenoble) 18 (1968), fasc. 2, 287-302.

[P] V. Ponomarev, Normal spaces as images of zero-dimensional ones, Soviet Math. Dokl. 1 (1960), 774-777.

[PT] J. R. Porter and J. D. Thomas, On ПI-closed and minimal Hausdorff spaces, Trans. Amer. Math. Soc. 138 (1969), 159-170.

[W] S. W. Willard, Functionally compact spaces, C-compact spaces and mappings of minimal Hausdorff spaces, Pacific J. Math. 38 (1971), 267-272.

Department of Mathematics, Washington and Lee University, Lexington, Virginia 24450

Department of Mathematics, Virginia Polytechnic Institute and State University, BlackSBURG, VirginIA 24061 